\title{
A New Approach to the Quadrature Rules with Gaussian Weights and Nodes
}

\author{
Giuliana Criscuolo ${ }^{1}$ and Salvatore Cuomo ${ }^{2, *}$ \\ ${ }^{1}$ Istituto per le Applicazioni del Calcolo "M. Picone" - CNR, Via P. Castellino 111, 80131 Napoli, Italy \\ 2 Dipartimento di Matematica e Applicazioni ”R. Caccioppoli”, Universtà degli Studi di Napoli Federico II, Via Chintia 80126 Napoli, \\ Italy
}

Received: 5 Aug. 2013, Revised: 2 Nov. 2013, Accepted: 3 Nov. 2013

Published online: 1 Sep. 2014

\begin{abstract}
To compute integrals on bounded or unbounded intervals we propose a new numerical approach by using weights and nodes of the classical Gauss quadrature rules. An account of the error and the convergence theory is given for the proposed quadrature formulas which have the advantage of reducing the condition number of the linear system arising when applying Nyström methods to solve integral equations. Numerical examples confirming the theoretical results are provided to illustrate the accuracy of the introduced method.
\end{abstract}

Keywords: Numerical integration, Quadrature rules, Gaussian weights and nodes. AMS subject classification: Primary 65D30; Secondary 65R20.

\section{Introduction}

Gauss quadrature rules represent an efficient numerical technique to compute definite integrals when the domain of integration is bounded or not. This justifies the continued interest to provide packages of efficient routines implementing the computation of the procedure to generate the parameters (i.e. weights and nodes) from which gaussian formulas depend (see [4], [5] and the references cited therein). In this paper we propose new quadrature formulas that depend on the same parameters typical of the classical gaussian formulas, so that the previous packages mentioned in the literature can be efficiently used for their computation. These new formulas consist essentially in a truncation of the classical gaussian formulas made omitting those nodes of the original formulas that are larger than a certain threshold. When the domain of integration is bounded, the proposed formulas, though converging, seem generally less accurate than the original gaussian formulas. However, they are also competitive with the latter ones with respect to the order of convergence when the integrand has a particular behavior, as well as being advantageous requiring a lower computational effort. In addition, the new formulas may be useful in other circumstances, such as when the integrand is a function taking significant values only in a very small part of the integration domain. In the case where the domain of integration is unbounded, the truncated formulas have a further advantage. In fact, we can show an error estimate that has already been proven for the original gaussian formulas but with more restrictive assumptions on the integrand [1]. The results in [1] and [6]-[11] for a related problem are specified and generalized here and in analogy with them, it turns out that a proper choice of the truncation point may result in an algorithm with smaller computational cost but identical or better error bounds.

Since the truncated formulas converge when the corresponding gaussian formulas converge, they may be profitably used as quadrature scheme of the Nyström method for solving integral equations. In fact, it will be possible to establish the stability, and thus the convergence, of the method which is characterized by a matrix of order less than that one of the matrix corresponding to the use of the original gaussian formulas. The investigation of this point has been extensively treated for truncated versions of the gaussian formulas which are special cases of those presented in the present paper (see [6]-[11]).

\footnotetext{
*Corresponding author e-mail: salvatore.cuomo@unina.it
} 
The remaining part of the paper is organized as follows. In Sections 2 and 3 we propose and discuss some convergence properties of the truncated gaussian formulas in the cases of bounded and unbounded domain of integrations, respectively. Finally, in Section 4, we present some numerical results and we give some computational considerations.

\section{Computation of integrals on bounded interval}

To compute the integral

$$
I\left(f w^{\alpha, \beta}\right)=\int_{-1}^{1} f(x) w^{\alpha, \beta}(x) d x,
$$

where $w^{\alpha, \beta}(x)=(1-x)^{\alpha}(1+x)^{\beta}, \alpha, \beta>-1$, we consider the classical Gauss-Jacobi quadrature rule

$$
\begin{array}{r}
I\left(f w^{\alpha, \beta}\right)=I_{n}^{G J}\left(f ; w^{\alpha, \beta}\right)+R_{n}^{G J}\left(f ; w^{\alpha, \beta}\right)= \\
\sum_{k=1}^{n} \lambda_{n, k}^{\alpha, \beta} f\left(x_{n, k}^{\alpha, \beta}\right)+R_{n}^{G J}\left(f ; w^{\alpha, \beta}\right),
\end{array}
$$

where $\lambda_{n, k}^{\alpha, \beta}, k=1, \ldots, n$ are the Christoffel constants with respect to the Jacobi weight $w^{\alpha, \beta}$, and $x_{n, k}^{\alpha, \beta}, k=1, \ldots, n$ are the zeros of the $n$th Jacobi orthogonal polynomial which we assume ordered in increasing order

$$
-1<x_{n, 1}^{\alpha, \beta}<x_{n, 2}^{\alpha, \beta}<\cdots<x_{n, n}^{\alpha, \beta}<1 .
$$

Let $\left\{\sigma_{n}\right\}_{n \in N}$ such that $\sigma_{n} \leq \sigma_{n+1}$ and $x_{n, 1}^{\alpha, \beta} \leq \sigma_{n} \leq x_{n, n}^{\alpha, \beta}$. We define the integer $c_{n}=c(n)$ as

$$
x_{n, c_{n}}^{\alpha, \beta} \leq \sigma_{n} \leq x_{n, c_{n}+1}^{\alpha, \beta}, \quad c_{n} \in\{1,2, \ldots, n-1\} .
$$

Consider the quadrature rule with gaussian weights and nodes

$$
\begin{array}{r}
I\left(f w^{\alpha, \beta}\right)=\widehat{I}_{n}^{G J}\left(f ; w^{\alpha, \beta}\right)+\widehat{R}_{n}^{G J}\left(f ; w^{\alpha, \beta}\right)= \\
\sum_{k=1}^{c_{n}} \lambda_{n, k}^{\alpha, \beta} f\left(x_{n, k}^{\alpha, \beta}\right)+\widehat{R}_{n}^{G J}\left(f ; w^{\alpha, \beta}\right) .
\end{array}
$$

This formula has degree of exactness 0 , however we will show that, for particular choices of the sequence $\left\{\sigma_{n}\right\}_{n \in N}$ and in particular assumptions about the integrand $f$, it is convergent like the original Gauss-Jacobi formula with the same order of convergence, while having a lower computational cost. In this regard the following theorem holds.

Theorem 1. Let $|f(x)| \leq K, x \in[1-\varepsilon, 1], \varepsilon>0, K \geq 0$. Then, for sufficiently large $n$,

$$
\begin{array}{r}
\left|\widehat{R}_{n}^{G J}\left(f ; w^{\alpha, \beta}\right)\right| \leq\left|R_{n}^{G J}\left(f ; w^{\alpha, \beta}\right)\right|+ \\
C_{1}\left(1-\sigma_{n}\right)^{\alpha+1} \max _{\sigma_{n} \leq x \leq 1}|f(x)|,
\end{array}
$$

where $C_{1}$ is independent of $n$ and $f$.
Proof. Since $\sigma_{n} \in[1-\varepsilon, 1]$, for sufficiently large $n$, we deduce

$$
\begin{array}{r}
\sum_{k=c_{n}+1}^{n} \lambda_{n, k}^{\alpha, \beta}\left|f\left(x_{n, k}^{\alpha, \beta}\right)\right| \leq \max _{\sigma_{n} \leq x \leq 1}|f(x)| \sum_{k=c_{n}+1}^{n} \lambda_{n, k}^{\alpha, \beta} \\
\leq \max _{\sigma_{n} \leq x \leq 1}|f(x)| \int_{c_{n}}^{1}(1-x)^{\alpha}(1+x)^{\beta} d x \\
\leq \max _{\sigma_{n} \leq x \leq 1}|f(x)| \max \left\{1,2^{\beta}\right\} \frac{\left(1-x_{n, c_{n}}^{\alpha, \beta}\right)^{\alpha+1}}{\alpha+1} \\
\leq C_{1} \max _{\sigma_{n} \leq x \leq 1}|f(x)|\left(1-\sigma_{n}\right)^{\alpha+1},
\end{array}
$$

where we have used the Markov-Stietjes inequalities and $1-x_{n, c_{n}}^{\alpha, \beta} \sim 1-\sigma_{n}{ }^{1}$. Then the assertion immediately follows taking into account that

$$
\widehat{R}_{n}^{G J}\left(f ; w^{\alpha, \beta}\right)=R_{n}^{G J}\left(f ; w^{\alpha, \beta}\right)+\sum_{k=c_{n}+1}^{n} \lambda_{n, k}^{\alpha, \beta} f\left(x_{n, k}^{\alpha, \beta}\right) .
$$

एा

We observe that, excluding the choice of a sequence $\left\{\sigma_{n}\right\}_{n \in N}$ definitively constant, having to be $\lim _{n \rightarrow \infty} \sigma_{n}=1$, it follows the convergence of the formula (2) equal to the convergence of the Gauss-Jacobi formula which has, however, a greater computational cost. About the order of convergence, the choice of the sequence $\left\{\sigma_{n}\right\}_{n \in N}$ plays a fundamental role. For example, if $f(x)=(1-x)^{v}, v \in R^{+}$ then, $\left|R_{n}^{G J}\left(f ; w^{\alpha, \beta}\right)\right| \leq$ const $n^{-\lfloor v\rfloor}$, where $\lfloor v\rfloor$ denotes the integer part of $v$, i.e. the largest integer less than or equal to $v$. On the other hand, $\left(1-\sigma_{n}\right)^{\alpha+1} \max _{\sigma_{n} \leq x \leq 1}|f(x)|=\left(1-\sigma_{n}\right)^{\alpha+1+v}=n^{-\alpha-1-v}$, having chosen $\sigma_{n}=1-n^{-1}$. Therefore, formula (2) is also competitive compared with the convergence order of the ordinary Gauss-Jacobi formula.

A consequence of the previous theorem is the following

Corollary 1. Let $\sigma_{n}=\sigma, n \in N,|\sigma|<1$, and $|f(x)| \leq K$, $x \in[\sigma, 1], K \geq 0$. Then, for sufficiently large $n$,

$$
\left|\widehat{R}_{n}^{G J}\left(f ; w^{\alpha, \beta}\right)\right| \leq\left|R_{n}^{G J}\left(f ; w^{\alpha, \beta}\right)\right|+C_{1}^{\prime} \max _{\sigma \leq x \leq 1}|f(x)|,
$$

where $C_{1}^{\prime}$ is independent of $n$ and $f$.

The previous corollary concerns the choice of a constant sequence $\sigma_{n}=\sigma, n \in N$, and it does not provide a convergence result, i.e. such a choice of the sequence does not lead to a convergent formula (2). However, when $f$ is significant with respect to the machine precision or to the required accuracy only in $[-1, \sigma],|\sigma|<1$, such a formula is of interest from a computational point of view. The formula (2) can also be interpreted as a formula to approximate the integral over the interval $\left[-1, \sigma_{n}\right]$. Indeed, the following convergence result holds.

\footnotetext{
1 If $A$ and $B$ are two expressions depending on some variables, then we write $A \sim B$ if and only if $\left|A B^{-1}\right|^{ \pm 1} \leq$ const uniformly for the variables under consideration.
} 
Theorem 2. Let $|f(x)| \leq K, x \in[1-\varepsilon, 1], \varepsilon>0, K \geq 0$. Then, for sufficiently large $n$,

$$
\begin{array}{r}
\left|\int_{-1}^{\sigma_{n}} f(x) w^{\alpha, \beta}(x) d x-\sum_{k=1}^{c_{n}} \lambda_{n, k}^{\alpha, \beta} f\left(x_{n, k}^{\alpha, \beta}\right)\right| \leq\left|R_{n}^{G J}\left(f ; w^{\alpha, \beta}\right)\right|+ \\
C_{1}^{\prime \prime}\left(1-\sigma_{n}\right)^{\alpha+1} \max _{\sigma_{n} \leq x \leq 1}|f(x)|,
\end{array}
$$

where $C_{1}^{\prime \prime}$ is independent of $n$ and $f$.

Proof. This result follows immediately from

$$
\begin{array}{r}
\left|\int_{-1}^{\sigma_{n}} f(x) w^{\alpha, \beta}(x) d x-\sum_{k=1}^{c_{n}} \lambda_{n, k}^{\alpha, \beta} f\left(x_{n, k}^{\alpha, \beta}\right)\right| \leq\left|\widehat{R}_{n}^{G J}\left(f ; w^{\alpha, \beta}\right)\right|+ \\
\left|\int_{\sigma_{n}}^{1} f(x) w^{\alpha, \beta}(x) d x\right| \leq\left|\widehat{R}_{n}^{G J}\left(f ; w^{\alpha, \beta}\right)\right|+ \\
\max _{\sigma_{n} \leq x \leq 1}|f(x)| \max \left\{1,2^{\beta}\right\} \frac{\left(1-\sigma_{n}\right)^{\alpha+1}}{\alpha+1},
\end{array}
$$

and using the Theorem 1 ए

The previous results may be specified if the integrand enjoys a particular monotony. In this regard, consider following results.

Theorem 3. Let $|f(x)| w^{\alpha, \beta}(x)$ be not increasing in $[1-$ $\varepsilon, 1], \varepsilon>0$. Then, for sufficiently large $n$,

$$
\begin{array}{r}
\left|\widehat{R}_{n}^{G J}\left(f ; w^{\alpha, \beta}\right)\right| \leq\left|R_{n}^{G J}\left(f ; w^{\alpha, \beta}\right)\right|+ \\
C_{2}\left\{\left\|f w^{\alpha, \beta}\right\|_{L_{1}\left[\sigma_{n}, 1\right]}+\frac{1}{n}\left|f\left(\sigma_{n}\right)\right| w^{\alpha+\frac{1}{2}, \beta+\frac{1}{2}}\left(\sigma_{n}\right)\right\},
\end{array}
$$

where $C_{2}$ is independent of $n$ and $f$.

Proof. Since $\sigma_{n} \in[1-\varepsilon, 1]$, for sufficiently large $n$, in view of the assumption on $f$, we have

$$
\begin{gathered}
\sum_{k=c_{n}+1}^{n} \lambda_{n, k}^{\alpha, \beta}\left|f\left(x_{n, k}^{\alpha, \beta}\right)\right|=\lambda_{n, c_{n}+1}^{\alpha, \beta}\left|f\left(x_{n, c_{n}+1}^{\alpha, \beta}\right)\right|+ \\
\sum_{k=c_{n}+2}^{n} \lambda_{n, k}^{\alpha, \beta}\left|f\left(x_{n, k}^{\alpha, \beta}\right)\right| \leq C_{2}\left\{\frac{1}{n}\left|f\left(\sigma_{n}\right)\right| w^{\alpha+\frac{1}{2}, \beta+\frac{1}{2}}\left(\sigma_{n}\right)\right. \\
\left.+\sum_{k=c_{n}+2}^{n}\left(x_{n, k}^{\alpha, \beta}-x_{n, k-1}^{\alpha, \beta}\right)\left|f\left(x_{n, k}^{\alpha, \beta}\right)\right| w^{\alpha, \beta}\left(x_{n, k}^{\alpha, \beta}\right)\right\} \leq \\
C_{2}\left\{\frac{1}{n}\left|f\left(\sigma_{n}\right)\right| w^{\alpha+\frac{1}{2}, \beta+\frac{1}{2}}\left(\sigma_{n}\right)+\left\|f w^{\alpha, \beta}\right\|_{L_{1}\left[\sigma_{n}, 1\right]}\right\},
\end{gathered}
$$

where we have used

$$
\begin{gathered}
\lambda_{n, k}^{\alpha, \beta} \sim \frac{1}{n}\left(1-x_{n, k}^{\alpha, \beta}\right)^{\alpha+\frac{1}{2}}+\left(1+x_{n, k}^{\alpha, \beta}\right)^{\beta+\frac{1}{2}}, \quad k=1, \ldots, n, \\
x_{n, k}^{\alpha, \beta}-x_{n, k-1}^{\alpha, \beta} \sim \frac{1}{n}\left(1-x_{n, k}^{\alpha, \beta}\right)^{\frac{1}{2}}\left(1+x_{n, k}^{\alpha, \beta}\right)^{\frac{1}{2}}, \quad k=1, \ldots, n-1
\end{gathered}
$$

(cfr. [13]), and $w^{\alpha, \beta}\left(x_{n, c_{n}+1}^{\alpha, \beta}\right) \sim w^{\alpha, \beta}\left(\sigma_{n}\right)$. Then the assertion immediately follows from (3). एा
Corollary 2. Let $\sigma_{n}=\sigma, n \in N,|\sigma|<1$, and let $|f(x)| w^{\alpha, \beta}(x)$ be not increasing in $[\sigma, 1]$. Then, for sufficiently large $n$,

$$
\begin{array}{r}
\left|\widehat{R}_{n}^{G J}\left(f ; w^{\alpha, \beta}\right)\right| \leq\left|R_{n}^{G J}\left(f ; w^{\alpha, \beta}\right)\right|+ \\
C_{2}^{\prime}\left\{\left\|f w^{\alpha, \beta}\right\|_{L_{1}[\sigma, 1]}+\frac{1}{n}|f(\sigma)| w^{\alpha+\frac{1}{2}, \beta+\frac{1}{2}}(\sigma)\right\},
\end{array}
$$

where $C_{2}^{\prime}$ is independent of $n$ and $f$.

Theorem 4. Let $|f(x)| w^{\alpha, \beta}(x)$ be not increasing in $[1-$ $\varepsilon, 1], \varepsilon>0$. Then, for sufficiently large $n$,

$$
\begin{gathered}
\left|\int_{-1}^{\sigma_{n}} f(x) w^{\alpha, \beta}(x) d x-\sum_{k=1}^{c_{n}} \lambda_{n, k}^{\alpha, \beta} f\left(x_{n, k}^{\alpha, \beta}\right)\right| \leq\left|R_{n}^{G J}\left(f ; w^{\alpha, \beta}\right)\right| \\
+C_{2}^{\prime \prime}\left\{\left\|f w^{\alpha, \beta}\right\|_{L_{1}\left[\sigma_{n}, 1\right]}+\frac{1}{n}\left|f\left(\sigma_{n}\right)\right| w^{\alpha+\frac{1}{2}, \beta+\frac{1}{2}}\left(\sigma_{n}\right)\right\},
\end{gathered}
$$

where $C_{2}^{\prime \prime}$ is independent of $n$ and $f$.

The previous theorems and corollaries are related to formulas of the type (2) which consist of a truncation of the Gauss-Jacobi formula at the nodes closest to the right extreme of the integration interval. In this case, the use of the truncation formula is of interest for functions that have a particular behavior in a neighborhood of this extreme. Same token can be constructed formulas that are well suited to the same situations in a neighborhood at the other extreme. Let $\left\{\sigma_{n}^{\prime}\right\}_{n \in N}$ such that $\sigma_{n}^{\prime} \geq \sigma_{n+1}^{\prime}$ and $x_{n, 1}^{\alpha, \beta} \leq \sigma_{n}^{\prime} \leq x_{n, n}^{\alpha, \beta}$. We define the integer $c_{n}^{\prime}=c^{\prime}(n)$ as

$$
x_{n, c_{n}^{\prime}}^{\alpha, \beta} \leq \sigma_{n}^{\prime} \leq x_{n, c_{n}^{\prime}+1}^{\alpha, \beta}, \quad c_{n}^{\prime} \in\{1,2, \ldots, n-1\} .
$$

Consider the quadrature rule with gaussian weights and nodes

$$
\begin{array}{r}
I\left(f w^{\alpha, \beta}\right)=\widetilde{I}_{n}^{G J}\left(f ; w^{\alpha, \beta}\right)+\widetilde{R}_{n}^{G J}\left(f ; w^{\alpha, \beta}\right)= \\
\sum_{k=c_{n}^{\prime}+1}^{c_{n}} \lambda_{n, k}^{\alpha, \beta} f\left(x_{n, k}^{\alpha, \beta}\right)+\widetilde{R}_{n}^{G J}\left(f ; w^{\alpha, \beta}\right),
\end{array}
$$

where the integer $c_{n}$ is defined in (1).

Regarding the latter formula may be for example set the following convergence results of which we omit the proof being quite similar to that of the Theorems 1 and 4 .

Theorem 5. Let $|f(x)| \leq K, x \in[-1,-1+\varepsilon] \cup[1-\varepsilon, 1]$, $\varepsilon>0, K \geq 0$. Then, for sufficiently large $n$,

$$
\begin{array}{r}
\left|\widetilde{R}_{n}^{G J}\left(f ; w^{\alpha, \beta}\right)\right| \leq\left|R_{n}^{G J}\left(f ; w^{\alpha, \beta}\right)\right|+ \\
C_{3}\left\{\left(1-\sigma_{n}\right)^{\alpha+1} \max _{\sigma_{n} \leq x \leq 1}|f(x)|+\left(1+\sigma_{n}^{\prime}\right)^{\beta+1} \max _{-1 \leq x \leq \sigma_{n}^{\prime}}|f(x)|\right\},
\end{array}
$$

where $C_{3}$ is independent of $n$ and $f$. 
Theorem 6. Let $|f(x)| w^{\alpha, \beta}(x)$ be not increasing in $[1-$ $\varepsilon, 1]$ and not decreasing in $[-1,-1+\varepsilon], \varepsilon>0$. Then, for sufficiently large $n$,

$$
\begin{aligned}
& \left|\widetilde{R}_{n}^{G J}\left(f ; w^{\alpha, \beta}\right)\right| \leq\left|R_{n}^{G J}\left(f ; w^{\alpha, \beta}\right)\right|+C_{4}\left\{\left\|f w^{\alpha, \beta}\right\|_{L_{1}\left[-1, \sigma_{n}^{\prime}\right] \cup\left[\sigma_{n}, 1\right]}\right. \\
& \left.+\frac{1}{n}\left|f\left(\sigma_{n}\right)\right| w^{\alpha+\frac{1}{2}, \beta+\frac{1}{2}}\left(\sigma_{n}\right)+\frac{1}{n}\left|f\left(\sigma_{n}^{\prime}\right)\right| w^{\alpha+\frac{1}{2}, \beta+\frac{1}{2}}\left(\sigma_{n}^{\prime}\right)\right\},
\end{aligned}
$$

where $C_{4}$ is independent of $n$ and $f$.

\section{Computation of integrals on unbounded interval}

The present section is devoted to the investigation of quadrature rules to compute the integral

$$
I\left(f w_{\gamma}\right)=\int_{0}^{\infty} f(x) w_{\gamma}(x) d x
$$

where $w_{\gamma}(x)=x^{\gamma} e^{-x}, \quad \gamma>-1$, and $f$ satisfies the smoothness condition if $r \geq 1$

$f \in W_{r}^{1}\left(w_{\gamma}\right):=\left\{f: f^{(r-1)} \in A C,\left\|f^{(r)} w_{\gamma+r / 2}\right\|_{1}<\infty\right\}$,

being $A C$ the set of all real functions which are absolutely continuous on any bounded subinterval of $(0, \infty)$.

We consider the classical Gauss-Laguerre quadrature rule

$$
\begin{gathered}
I\left(f w_{\gamma}\right)=I_{n}^{G L}\left(f ; w_{\gamma}\right)+R_{n}^{G L}\left(f ; w_{\gamma}\right)= \\
\sum_{k=1}^{n} \lambda_{n, k}^{\gamma} f\left(x_{n, k}^{\gamma}\right)+R_{n}^{G L}\left(f ; w_{\gamma}\right),
\end{gathered}
$$

where $\lambda_{n, k}^{\gamma}, k=1, \ldots, n$ are the Christoffel constants with respect to the generalized Laguerre weight $w_{\gamma}$, and $x_{n, k}^{\gamma}$, $k=1, \ldots, n$ are the zeros of the $n$th generalized Laguerre orthogonal polynomial ordered in increasing order.

$$
\frac{c_{1}}{n}<x_{n, 1}^{\gamma}<x_{n, 2}^{\gamma}<\ldots<x_{n, n}^{\gamma}<4 n-2 \gamma+2-c_{2}(4 n)^{\frac{1}{3}}
$$

with some constants $c_{1}$ and $c_{2}$ independent of $n \geq 1$ and $k \in\{1,2, \ldots, n\}$, (see [3] and [13]).

Let $\left\{\tau_{n}\right\}_{n \in N}$ such that $\tau_{n} \leq \tau_{n+1}, 0<\tau_{n} \leq c n, 0<c<1$, and $\lim _{n \rightarrow \infty} \tau_{n}=\infty$. We define the integer $c_{n}=c(n)$ as

$$
x_{n, c_{n}}^{\gamma}=\min _{1 \leq k \leq n}\left\{x_{n, k}^{\gamma}: x_{n, k}^{\gamma} \geq 4 \tau_{n}\right\} .
$$

Consider the quadrature rule with gaussian weights and nodes

$$
\begin{array}{r}
I\left(f w_{\gamma}\right)=\widehat{I}_{n}^{G L}\left(f ; w_{\gamma}\right)+\widehat{R}_{n}^{G L}\left(f ; w_{\gamma}\right)= \\
\sum_{k=1}^{c_{n}} \lambda_{n, k}^{\gamma} f\left(x_{n, k}^{\gamma}\right)+\widehat{R}_{n}^{G L}\left(f ; w_{\gamma}\right) .
\end{array}
$$

Define

$$
\delta_{c_{n}}(x)=\delta\left(\frac{x-x_{n, c_{n}}^{\gamma}}{x_{n, c_{n}+1}^{\gamma}-x_{n, c_{n}}^{\gamma}}\right),
$$

where $\delta \in C^{\infty}(\mathbf{R}), \delta(x)=0$ when $x \leq 0$ and $\delta(x)=1$ when $x \geq 1$.

We remark that the function

$$
f_{c_{n}}=f-\delta_{c_{n}} f
$$

has the same degree of smoothness of $f$.

The following lemmas are needed to prove the convergence of the quadrature rule (5).

Lemma 1. Let $N_{n}=\left[\frac{1}{c+1} \tau_{n}\right]$ with $0<c<1$ fixed. For any $f \in W_{1}^{1}\left(w_{\gamma}\right)$, we have

$\left\|\left(f-f_{c_{n}}\right) w_{\gamma}\right\|_{1} \leq \bar{C}_{1}\left[\frac{E_{N_{n}-1}\left(f^{\prime}\right)_{w_{\gamma+1 / 2}, 1}}{\sqrt{N_{n}}}+e^{-\bar{C}_{2} N_{n}}\left\|f w_{\gamma}\right\|_{1}\right]$,

where $E_{N_{n}-1}\left(f^{\prime}\right)_{w_{\gamma+1 / 2}, 1}=\inf _{P \in \Pi_{N_{n-1}}}\left\|\left(f^{\prime}-P\right) w_{\gamma+1 / 2}\right\|_{1},^{2}$ and $\bar{C}_{1}, \bar{C}_{2}$ are independent of $n$ and $f$.

Proof. The proof is based on the inequality

$$
\begin{gathered}
\int_{2(1+\sigma) v}^{\infty}\left|p_{v}(x)\right| w_{\gamma}(x) d x \leq \\
A e^{-C v} \int_{0}^{\infty}\left|p_{v}(x)\right| w_{\gamma}(x) d x, \quad \sigma>0,
\end{gathered}
$$

for any polynomial $p_{v}$ of degree $v>2(2 \gamma+1) / \sigma$ and where $A=A(\sigma)$ and $C=C(\sigma)$ are independent of $v$ and $p_{v},($ see $[2])$.

By using (3) we have

$$
\left\|\left(f-f_{c_{n}}\right) w_{\gamma}\right\|_{1} \leq\left\|f w_{\gamma}\right\|_{L^{1}\left[4 \tau_{n}, \infty\right)} \leq E_{N_{n}}(f)_{w_{\gamma}, 1}+
$$

$$
\bar{C} e^{-\bar{C}_{2} N_{n}}\left\|f w_{\gamma}\right\|_{1},
$$

where $E_{N_{n}}(f)_{w_{\gamma}, 1}=\inf _{P \in \Pi_{N_{n}}}\left\|(f-P) w_{\gamma}\right\|_{1}$, and $\bar{C}, \bar{C}_{2}$ independent of $n$ and $f$.

Thus, the assertion follows by Favard's theorem. ए

Lemma 2. Let $N_{n}=\left[\frac{1}{c+1} \tau_{n}\right]$ with $0<c<1$ fixed. For any $f \in W_{1}^{1}\left(w_{\gamma}\right)$, we have

$\left|R_{n}^{G L}\left(f_{c_{n}} w_{\gamma}\right)\right| \leq \tilde{C}_{1}\left[\frac{E_{N_{n}-1}\left(f^{\prime}\right)_{w_{\gamma+1 / 2}, 1}}{\sqrt{N_{n}}}+e^{-\tilde{C}_{2} N_{n}}\left\|f w_{\gamma}\right\|_{1}\right]$

where $E_{N_{n}-1}\left(f^{\prime}\right)_{w_{\gamma+1 / 2}, 1}=\inf _{P \in \Pi_{N_{n}-1}}\left\|\left(f^{\prime}-P\right) w_{\gamma+1 / 2}\right\|_{1}$, and $\tilde{C}_{1}, \tilde{C}_{2}$ are independent of $n$ and $f$.

$2 \quad \Pi_{n}$ denotes the set of the polynomials of degree $\leq n$. 
Proof. By using Peano's theorem [12], we have

$$
R_{n}^{G L}\left(f_{c_{n}} ; w_{\gamma}\right)=\int_{0}^{\infty} R_{n}^{G L}\left((.-t)_{+}^{0} ; w_{\gamma}\right) f_{c_{n}}^{\prime}(t) d t
$$

where $(x-t)_{+}^{0}=1$ if $t<x$ and $(x-t)_{+}^{0}=0$ if $t \geq x$.

For $0 \leq t \leq x_{n, 1}^{\gamma}$,

$$
\begin{aligned}
& \left|R_{n}^{G L}\left((.-t)_{+}^{0} ; w_{\gamma}\right)\right|=\mid \int_{0}^{\infty}(x-t)_{+}^{0} w_{\gamma}(x) d x- \\
& \sum_{k=1}^{n} \lambda_{n, k}^{\gamma}\left(x_{n, k}^{\gamma}-t\right)_{+}^{0}||-\int_{0}^{t} w_{\gamma}(x) d x \mid \leq \frac{C^{\prime}}{n} w_{\gamma+\frac{1}{2}}(t),
\end{aligned}
$$

where $C^{\prime}$ is independent of $n$.

For $t>x_{n, 1}^{\gamma}$, being

$$
\left|R_{n}^{G L}\left((.-t)_{+}^{0} ; w_{\gamma}\right)\right| \leq \lambda_{n}^{\gamma}(t)
$$

where $\lambda_{n}^{\gamma}(t)$ is the $n$th Christoffel function with respect to the weight $w_{\gamma}$, we deduce

$$
\begin{aligned}
\left|R_{n}^{G L}\left(f_{c_{n}} ; w_{\gamma}\right)\right| & \leq \int_{0}^{x_{n, c_{n}+1}^{\gamma}}\left|R_{n}^{G L}\left((.-t)_{+}^{0} ; w_{\gamma}\right)\right|\left|f_{c_{n}}^{\prime}(t)\right| d t \\
& \leq \frac{\bar{C}}{\sqrt{n}} \int_{0}^{x_{n, c_{n}+1}^{\gamma}}\left|f_{c_{n}}^{\prime}(x)\right| w_{\gamma+\frac{1}{2}}(x) d x,
\end{aligned}
$$

having used

$\lambda_{n}^{\gamma}(t) \leq \bar{C} \sqrt{n} w_{\gamma+\frac{1}{2}}(t), \quad x_{n, 1}^{\gamma} \leq t \leq 4 c n, n \geq 1,0<c<1$,

(see [3] and [13]).

Thus, since $\delta_{c_{n}}(x)=0$ if $x \leq x_{n, c_{n}}^{\gamma}$, and $\delta_{c_{n}}^{\prime}(x) \leq\left\|\delta_{c_{n}}^{\prime}\right\|_{\infty} /\left(x_{n, c_{n}+1}^{\gamma}-x_{n, c_{n}}^{\gamma}\right) \sim \frac{\sqrt{n}}{x_{n, c_{n}}^{\gamma}}$ for $x \in\left(x_{n, c_{n}}^{\gamma}, x_{n, c_{n}+1}^{\gamma}\right)$, where we have used $x_{n, k}^{\gamma}-x_{n, k-1}^{\gamma} \sim \sqrt{x_{n, k}^{\gamma} /\left(4 n-x_{n, k}^{\gamma}\right)}, k \in\{2,3, \ldots, n\}$, (see [3]), recalling that $f_{c_{n}}=f-\delta_{c_{n}} f$, we can write

$$
\left|R_{n}^{G L}\left(f_{c_{n}} ; w^{\gamma}\right)\right| \leq C^{\prime \prime}\left[\frac{\left\|f^{\prime} w_{\gamma+\frac{1}{2}}\right\|_{1}}{\sqrt{n}}+\left\|f w_{\gamma}\right\|_{L^{1}\left[4 \tau_{n}, \infty\right)}\right],
$$

with some constant $C^{\prime \prime}$ independent of $n$ and $f$.

Then, by (3) and Favard's theorem, the assertion follows एा

Theorem 7. For any $f \in W_{1}^{1}\left(w_{\gamma}\right)$ we have, for sufficiently large $n$,

$\left|\widehat{R}_{n}^{G L}\left(f ; w_{\gamma}\right)\right| \leq \hat{C}_{1}\left[\frac{E_{N_{n}-1}\left(f^{\prime}\right)_{w_{\gamma+1 / 2}, 1}}{\sqrt{N_{n}}}+e^{-\hat{C}_{2} N_{n}}\left\|f w_{\gamma}\right\|_{1}\right]$,

where

$$
E_{N_{n}-1}\left(f^{\prime}\right)_{w_{\gamma+1 / 2}, 1}=
$$

$\inf _{P \in \Pi_{N_{n}-1}}\left\|\left(f^{\prime}-P\right) w_{\gamma+1 / 2}\right\|_{1}, N_{n}=\left[\frac{1}{c+1} \tau_{n}\right], 0<c<1$, and $\hat{C}_{1}, \hat{C}_{2}$ are independent of $n$ and $f$.
Proof. The assertion immediately follows from Lemmas 1 and taking into account that

$$
\widehat{R}_{n}^{G L}\left(f ; w_{\gamma}\right)=R_{n}^{G L}\left(f_{c_{n}} ; w_{\gamma}\right)+\left\|\left(f-f_{c_{n}}\right) w_{\gamma}\right\|_{1} .
$$

एा

We observe that, unlike what happens in the case of the bounded interval, the formula (5) performs better than the ordinary Gauss-Laguerre rule. Indeed, it was recently shown the same estimate of Theorem 7 for the gaussian error $R_{n}^{G L}\left(f ; w_{\gamma}\right)$ but only in more restrictive assumptions on the function $\mathrm{f}$ (see Corollary 1 in [1]).

Since the Christoffel constants $\lambda_{n, k}^{\gamma}$ decrease rapidly with $k$ goes to $n$, it happens that for sufficiently large $k$ the quantities $\lambda_{n, k}^{\gamma} f\left(x_{n, k}^{\gamma}\right)$ are not significant with respect to the machine precision. In this case the following formula is useful in computations

$$
\begin{array}{r}
I\left(f w_{\gamma}\right)=\bar{I}_{n}^{G L}\left(f ; w_{\gamma}\right)+\bar{R}_{n}^{G L}\left(f ; w_{\gamma}\right) \\
=\sum_{x_{n, k}^{\gamma} \leq \tau} \lambda_{n, k}^{\gamma} f\left(x_{n, k}^{\gamma}\right)+\bar{R}_{n}^{G L}\left(f ; w_{\gamma}\right),
\end{array}
$$

where $\tau>0$ is fixed in a suitable way. In this regard the following theorem holds.

Theorem 8. For any $f \in W_{1}^{1}\left(w_{\gamma}\right)$ we have, for sufficiently large $n$,

$$
\begin{array}{r}
\left|\bar{R}_{n}^{G L}\left(f ; w_{\gamma}\right)\right| \leq \hat{C}_{1}\left[\frac{E_{N_{n}-1}\left(f^{\prime}\right)_{w_{\gamma+1 / 2}, 1}}{\sqrt{N_{n}}}+e^{-\hat{C}_{2} N_{n}}\left\|f w_{\gamma}\right\|_{1}\right]+ \\
\bar{C}_{3} n^{3 / 2} \max _{x \geq \tau}\left|f(x) w_{\gamma+\frac{1}{2}}(x)\right|,
\end{array}
$$

where $E_{N_{n}-1}\left(f^{\prime}\right)_{w_{\gamma+1 / 2}, 1}=\inf _{P \in \Pi_{N_{n}-1}}\left\|\left(f^{\prime}-P\right) w_{\gamma+1 / 2}\right\|_{1}$, $N_{n}=\left[\frac{1}{c+1} \tau_{n}\right], 0<c<1$, and $\hat{C}_{1}, \hat{C}_{2}, \bar{C}_{3}$ are independent of $n$ and $f$.

Proof. The assertion immediately follows from Theorem 7 taking into account that

$$
\bar{R}_{n}^{G L}\left(f ; w_{\gamma}\right)=\hat{R}_{n}^{G L}\left(f ; w_{\gamma}\right)-\sum_{x_{n, k}^{\gamma}>\tau}^{c_{n}} \lambda_{n, k}^{\gamma} f\left(x_{n, k}^{\gamma}\right),
$$

and using (7). एा

Finally, it is interesting to truncate the Gauss-Laguerre formula also with regard nodes close to 0 when the function $f$ has a particular behavior near this point. Let $\left\{\tau_{n}^{\prime}\right\}_{n \in N}$ such that $\tau_{n}^{\prime} \geq \tau_{n+1}^{\prime}$ and $x_{n, 1}^{\gamma} \leq \tau_{n}^{\prime} \leq x_{n, n}^{\gamma}$. We define the integer $v_{n}=v(n)$ as

$$
x_{n, v_{n}}^{\gamma} \leq \tau_{n}^{\prime} \leq x_{n, v_{n}+1}^{\gamma}, \quad v_{n} \in\{1,2, \ldots, n-1\} .
$$

Consider the quadrature rule with gaussian weights and nodes

$$
\begin{array}{r}
I\left(f w_{\gamma}\right)=\widetilde{I}_{n}^{G J}\left(f ; w_{\gamma}\right)+\widetilde{R}_{n}^{G J}\left(f ; w_{\gamma}\right)= \\
\sum_{k=v_{n}+1}^{c_{n}} \lambda_{n, k}^{\gamma} f\left(x_{n, k}^{\gamma}\right)+\widetilde{R}_{n}^{G J}\left(f ; w_{\gamma}\right),
\end{array}
$$


Table 1: Relative errors for the example $\int_{-1}^{1} f_{1}(x) d x$

\begin{tabular}{|r|lr|lr|lr|}
\hline$n$ & $\sigma_{n}=1-\frac{1}{n}$ & & \multicolumn{2}{|l|}{$\sigma_{n}=1-\frac{1}{\sqrt{n}}$} & & \multicolumn{3}{|l|}{$\sigma_{n}=1-\frac{1}{\sqrt[4]{n}}$} & \\
\hline 4 & $7.4981 \mathrm{e}-05$ & {$[1]$} & $7.4981 \mathrm{e}-05$ & {$[1]$} & 0.0304 & {$[2]$} \\
8 & $2.6413 \mathrm{e}-07$ & {$[1]$} & $1.6792 \mathrm{e}-04$ & {$[2]$} & 0.0048 & {$[3]$} \\
16 & $4.9695 \mathrm{e}-07$ & {$[2]$} & $1.9670 \mathrm{e}-04$ & {$[4]$} & 0.0012 & {$[5]$} \\
32 & $1.1580 \mathrm{e}-09$ & {$[2]$} & $1.7033 \mathrm{e}-05$ & {$[6]$} & 0.0012 & {$[10]$} \\
64 & $9.1447 \mathrm{e}-11$ & {$[3]$} & $3.3379 \mathrm{e}-06$ & {$[10]$} & $4.9848 \mathrm{e}-04$ & {$[18]$} \\
128 & $1.6519 \mathrm{e}-11$ & {$[5]$} & $7.6378 \mathrm{e}-07$ & {$[17]$} & $1.8150 \mathrm{e}-04$ & {$[32]$} \\
256 & $6.3112 \mathrm{e}-13$ & {$[7]$} & $1.8033 \mathrm{e}-07$ & {$[29]$} & $8.9335 \mathrm{e}-05$ & {$[59]$} \\
512 & $6.6592 \mathrm{e}-14$ & {$[11]$} & $3.2698 \mathrm{e}-08$ & {$[48]$} & $3.8077 \mathrm{e}-05$ & {$[107]$} \\
\hline
\end{tabular}

Table 2: Relative errors for the example $\int_{-1}^{1} f_{2}(x) d x$

\begin{tabular}{|r|rr|lr|lr|}
\hline$n$ & $\sigma_{n}=1-\frac{1}{n}$ & & \multicolumn{2}{|c|}{$\sigma_{n}=1-\frac{1}{\sqrt{n}}$} & & \multicolumn{2}{|l|}{$\sigma_{n}=1-\frac{1}{\sqrt[4]{n}} r$} \\
\hline 4 & $3.7802 \mathrm{e}-06$ & {$[1]$} & $3.7802 \mathrm{e}-06$ & {$[1]$} & 0.0048 & {$[2]$} \\
8 & $3.6473 \mathrm{e}-10$ & {$[1]$} & $2.5034 \mathrm{e}-06$ & {$[2]$} & $3.7565 \mathrm{e}-04$ & {$[3]$} \\
16 & $5.5056 \mathrm{e}-10$ & {$[2]$} & $3.9856 \mathrm{e}-06$ & {$[4]$} & $5.8093 \mathrm{e}-05$ & {$[5]$} \\
32 & $8.7003 \mathrm{e}-14$ & {$[2]$} & $1.2285 \mathrm{e}-07$ & {$[6]$} & $6.0245 \mathrm{e}-05$ & {$[10]$} \\
64 & $3.4444 \mathrm{e}-15$ & {$[3]$} & $1.2048 \mathrm{e}-08$ & {$[10]$} & $1.6884 \mathrm{e}-05$ & {$[18]$} \\
128 & $2.5514 \mathrm{e}-16$ & {$[5]$} & $1.4495 \mathrm{e}-09$ & {$[17]$} & $3.9386 \mathrm{e}-06$ & {$[32]$} \\
256 & $3.8271 \mathrm{e}-16$ & {$[7]$} & $1.8101 \mathrm{e}-10$ & {$[29]$} & $1.4165 \mathrm{e}-06$ & {$[59]$} \\
512 & $7.6542 \mathrm{e}-16$ & {$[11]$} & $1.5391 \mathrm{e}-11$ & {$[48]$} & $4.1346 \mathrm{e}-07$ & {$[107]$} \\
\hline
\end{tabular}

Table 3: Relative errors for the example $\int_{-1}^{1} f_{3}(x) d x$

\begin{tabular}{|r|lr|lr|lr|}
\hline$n$ & $\sigma_{n}=1-\frac{1}{n}$ & & \multicolumn{2}{|l|}{$\sigma_{n}=1-\frac{1}{\sqrt{n}}$} & & \multicolumn{2}{|l|}{$\sigma_{n}=1-\frac{1}{\sqrt[4]{n}}$} & \\
\hline 4 & 0.1235 & {$[1]$} & 0.1235 & {$[1]$} & 0.1235 & {$[2]$} \\
8 & $3.8490 \mathrm{e}-05$ & {$[1]$} & $3.8525 \mathrm{e}-05$ & {$[2]$} & $3.9268 \mathrm{e}-05$ & {$[3]$} \\
16 & $2.8557 \mathrm{e}-09$ & {$[2]$} & $4.0022 \mathrm{e}-08$ & {$[4]$} & $1.8081 \mathrm{e}-07$ & {$[5]$} \\
32 & $5.3603 \mathrm{e}-10$ & {$[2]$} & $9.8657 \mathrm{e}-09$ & {$[6]$} & $1.8190 \mathrm{e}-07$ & {$[10]$} \\
64 & $2.7235 \mathrm{e}-10$ & {$[3]$} & $4.9043 \mathrm{e}-09$ & {$[10]$} & $8.0557 \mathrm{e}-08$ & {$[18]$} \\
128 & $1.7624 \mathrm{e}-10$ & {$[5]$} & $2.8863 \mathrm{e}-09$ & {$[17]$} & $3.7288 \mathrm{e}-08$ & {$[32]$} \\
256 & $8.2728 \mathrm{e}-11$ & {$[7]$} & $1.8274 \mathrm{e}-09$ & {$[29]$} & $2.3537 \mathrm{e}-08$ & {$[59]$} \\
512 & $4.9556 \mathrm{e}-11$ & {$[11]$} & $1.1207 \mathrm{e}-09$ & {$[48]$} & $1.4509 \mathrm{e}-08$ & {$[107]$} \\
\hline
\end{tabular}

Table 4: Relative errors for the example $\int_{-1}^{1} f_{4}(x) d x$

\begin{tabular}{|r|lr|lr|lr|}
\hline$n$ & $\sigma_{n}=1-\frac{1}{n}$ & & \multicolumn{2}{|l|}{$\sigma_{n}=1-\frac{1}{\sqrt{n}}$} & & \multicolumn{2}{|l|}{$\sigma_{n}=1-\frac{1}{\sqrt[4]{n}}$} & \\
\hline 4 & 0.5672 & {$[1]$} & 0.5672 & {$[1]$} & 0.5672 & {$[2]$} \\
8 & 0.0083 & {$[1]$} & 0.0083 & {$[2]$} & 0.0083 & {$[3]$} \\
16 & $8.1887 \mathrm{e}-10$ & {$[2]$} & $8.1887 \mathrm{e}-10$ & {$[4]$} & $8.1889 \mathrm{e}-10$ & {$[5]$} \\
32 & $1.9533 \mathrm{e}-15$ & {$[2]$} & $2.1035 \mathrm{e}-15$ & {$[6]$} & $3.4258 \mathrm{e}-14$ & {$[10]$} \\
64 & $6.3106 \mathrm{e}-15$ & {$[3]$} & $6.3106 \mathrm{e}-15$ & {$[10]$} & $1.2922 \mathrm{e}-14$ & {$[18]$} \\
128 & $1.5025 \mathrm{e}-15$ & {$[5]$} & $1.5025 \mathrm{e}-15$ & {$[17]$} & $1.5025 \mathrm{e}-15$ & {$[32]$} \\
256 & $1.0668 \mathrm{e}-14$ & {$[7]$} & $1.0668 \mathrm{e}-14$ & {$[29]$} & $1.0968 \mathrm{e}-14$ & {$[59]$} \\
512 & $3.0050 \mathrm{e}-16$ & {$[11]$} & $3.0050 \mathrm{e}-16$ & {$[48]$} & $3.0050 \mathrm{e}-16$ & {$[107]$} \\
\hline
\end{tabular}




Table 5: Relative errors for the example $\int_{-1}^{\sigma_{n}} f_{2}(x) d x$
\begin{tabular}{|r|rr|lr|lrr|}
\hline$n$ & $\sigma_{n}=1-\frac{1}{n}$ & & \multicolumn{2}{|l|}{$\sigma_{n}=1-\frac{1}{\sqrt{n}}$} & & $\sigma_{n}=1-\frac{1}{\sqrt[4]{n}}$ & \\
\hline 4 & $2.4315 \mathrm{e}-06$ & {$[1]$} & $1.1830 \mathrm{e}-04$ & {$[1]$} & 0.0036 & {$[2]$} \\
8 & $1.4536 \mathrm{e}-08$ & {$[1]$} & $1.0328 \mathrm{e}-05$ & {$[2]$} & $8.6781 \mathrm{e}-07$ & {$[3]$} \\
16 & $3.8593 \mathrm{e}-10$ & {$[2]$} & $2.6369 \mathrm{e}-06$ & {$[4]$} & $6.3985 \mathrm{e}-05$ & {$[5]$} \\
32 & $1.7319 \mathrm{e}-12$ & {$[2]$} & $1.8910 \mathrm{e}-08$ & {$[6]$} & $2.0670 \mathrm{e}-05$ & {$[10]$} \\
64 & $1.6584 \mathrm{e}-14$ & {$[3]$} & $2.8534 \mathrm{e}-09$ & {$[10]$} & $4.0532 \mathrm{e}-06$ & {$[18]$} \\
128 & $3.8271 \mathrm{e}-16$ & {$[5]$} & $1.6778 \mathrm{e}-10$ & {$[17]$} & $2.2132 \mathrm{e}-07$ & {$[32]$} \\
256 & $3.8271 \mathrm{e}-16$ & {$[7]$} & $1.6370 \mathrm{e}-11$ & {$[29]$} & $6.7839 \mathrm{e}-08$ & {$[59]$} \\
512 & $7.6542 \mathrm{e}-16$ & {$[11]$} & $1.9142 \mathrm{e}-12$ & {$[48]$} & $2.3799 \mathrm{e}-08$ & {$[107]$} \\
\hline
\end{tabular}

\begin{tabular}{|} 
Table 6: Relative errors for the example $\int_{-1}^{\sigma_{n}} f_{3}(x) d x$ \\
\begin{tabular}{|r|rr|lr|lrr|}
\hline$n$ & $\sigma_{n}=1-\frac{1}{n}$ & & \multicolumn{2}{|l|}{$\sigma_{n}=1-\frac{1}{\sqrt{n}}$} & & $\sigma_{n}=1-\frac{1}{\sqrt[4]{n}}$ & \\
\hline 4 & 0.1235 & {$[1]$} & 0.1235 & {$[1]$} & 0.1235 & {$[2]$} \\
8 & $3.8485 \mathrm{e}-05$ & {$[1]$} & $3.8456 \mathrm{e}-05$ & {$[2]$} & $3.8482 \mathrm{e}-05$ & {$[3]$} \\
16 & $1.0661 \mathrm{e}-09$ & {$[2]$} & $1.6973 \mathrm{e}-08$ & {$[4]$} & $1.2303 \mathrm{e}-07$ & {$[5]$} \\
32 & $2.2008 \mathrm{e}-10$ & {$[2]$} & $1.4686 \mathrm{e}-10$ & {$[6]$} & $4.5893 \mathrm{e}-08$ & {$[10]$} \\
64 & $7.6228 \mathrm{e}-11$ & {$[3]$} & $2.2868 \mathrm{e}-10$ & {$[10]$} & $1.1893 \mathrm{e}-08$ & {$[18]$} \\
128 & $8.7567 \mathrm{e}-12$ & {$[5]$} & $4.1109 \mathrm{e}-11$ & {$[17]$} & $9.4843 \mathrm{e}-10$ & {$[32]$} \\
256 & $6.2114 \mathrm{e}-13$ & {$[7]$} & $3.7809 \mathrm{e}-11$ & {$[29]$} & $4.8862 \mathrm{e}-10$ & {$[59]$} \\
512 & $8.9031 \mathrm{e}-12$ & {$[11]$} & $2.4744 \mathrm{e}-11$ & {$[48]$} & $2.9971 \mathrm{e}-10$ & {$[107]$} \\
\hline
\end{tabular}
\end{tabular}

Table 7: Relative errors for the example $\int_{0}^{\infty} f_{5}(x) e^{-x} d x$

\begin{tabular}{|r|cr|cr|cr|cr|}
\hline$n$ & $\tau_{n}^{\prime}=\frac{1}{\sqrt{n}}$ & $\tau_{n}=\frac{n}{2}$ & $\tau_{n}^{\prime}=\frac{1}{\sqrt[4]{n}}$ & $\tau_{n}=\frac{n}{2}$ & $\tau_{n}^{\prime}=\frac{1}{\sqrt{n}}$ & $\tau_{n}=2 \sqrt{n}$ & $\tau_{n}^{\prime}=\frac{1}{\sqrt[4]{n}}$ & $\tau_{n}=2 \sqrt{n}$ \\
\hline 8 & $3.0304 \mathrm{e}-04$ & {$[0,1]$} & $3.0304 \mathrm{e}-04$ & {$[1,1]$} & 0.4372 & {$[0,4]$} & 0.4372 & {$[1,4]$} \\
16 & $1.8517 \mathrm{e}-07$ & {$[0,3]$} & $2.3808 \mathrm{e}-07$ & {$[2,3]$} & 0.0546 & {$[0,9]$} & 0.0546 & {$[2,9]$} \\
32 & $6.4162 \mathrm{e}-16$ & {$[1,6]$} & $4.7290 \mathrm{e}-11$ & {$[2,6]$} & 0.0012 & {$[1,20]$} & 0.0012 & {$[2,20]$} \\
64 & $3.2979 \mathrm{e}-14$ & {$[2,12]$} & $2.8329 \mathrm{e}-10$ & {$[3,12]$} & $4.4579 \mathrm{e}-06$ & {$[2,44]$} & $4.4582 \mathrm{e}-06$ & {$[3,44]$} \\
128 & $6.4162 \mathrm{e}-16$ & {$[2,23]$} & $9.9652 \mathrm{e}-11$ & {$[4,23]$} & $1.6115 \mathrm{e}-10$ & {$[2,94]$} & $2.6080 \mathrm{e}-10$ & {$[4,94]$} \\
256 & $1.0266 \mathrm{e}-15$ & {$[2,47]$} & $7.9062 \mathrm{e}-12$ & {$[5,47]$} & $1.0266 \mathrm{e}-15$ & {$[2,135]$} & $7.9062 \mathrm{e}-12$ & {$[5,135]$} \\
512 & $3.8497 \mathrm{e}-16$ & {$[3,93]$} & $2.4433 \mathrm{e}-13$ & {$[6,93]$} & $3.8497 \mathrm{e}-16$ & {$[3,416]$} & $2.4433 \mathrm{e}-13$ & {$[6,416]$} \\
\hline
\end{tabular}

where the integer $c_{n}$ is defined in (4). Regarding the latter formula may be set the following convergence result of which we omit the proof being quite similar to that of the Theorems 1 and 7 .

Theorem 9. For any $f \in W_{1}^{1}\left(w_{\gamma}\right)$ such that $|f(x)| \leq K$, $x \in[0, \varepsilon], \varepsilon>0$ and $K \geq 0$ we have, for sufficiently large $n$,

$$
\begin{array}{r}
\left|\widetilde{R}_{n}^{G L}\left(f ; w_{\gamma}\right)\right| \leq \hat{C}_{1}\left[\frac{E_{N_{n}-1}\left(f^{\prime}\right)_{w_{\gamma+1 / 2}, 1}}{\sqrt{N_{n}}}+e^{-\hat{C}_{2} N_{n}}\left\|f w_{\gamma}\right\|_{1}\right]+ \\
\bar{C}_{4}\left(\tau_{n}^{\prime}\right)^{\gamma+1} \max _{0 \leq x \leq \tau_{n}^{\prime}}|f(x)|,
\end{array}
$$

where $E_{N_{n}-1}\left(f^{\prime}\right)_{w_{\gamma+1 / 2}, 1}=\inf _{P \in \Pi_{N_{n}-1}}\left\|\left(f^{\prime}-P\right) w_{\gamma+1 / 2}\right\|_{1}$, $N_{n}=\left[\frac{1}{c+1} \tau_{n}\right], 0<c<1$, and $\hat{C}_{1}, \hat{C}_{2}, \bar{C}_{4}$ are independent of $n$ and $f$.

\section{Numerical results}

A set of test examples are considered to confirm numerically the convergence of the formula (2) in agreement with the main result consisting in the Theorem 1 and in the subsequent remarks. We have computed the integrals

$$
I\left(f_{i} w^{\alpha, \beta}\right)=\int_{-1}^{1} f_{i}(x) w^{\alpha, \beta}(x) d x, \quad i \in\{1,2,3,4\},
$$


assuming $\alpha=\beta=0$ and $f_{i}(x)=(1-x)^{v_{i}}, i \in\{1,2\}$, $v_{1}=7 / 2, v_{2}=11 / 2, f_{i}(x)=\exp \left(2-a_{i} \frac{x+1}{2}\right), i \in\{3,4\}$, $a_{3}=20, a_{4}=40$. Obviously, these integrals can be efficiently computed by the gaussian rule with respect to a suitable weight, but our choice is merely demonstrative of the above. We have obtained the results for the relative errors $\left|\widehat{R}_{n}^{G J}\left(f_{i} ; w^{0,0}\right)\right| /\left|I\left(f_{i} w^{0,0}\right)\right|, i \in\{1,2,3,4\}$ reported in Tables 1-4 for different choices of $\left\{\sigma_{n}\right\}_{n \in N}$, where $n$ is the number of the gaussian nodes while the number in square brackets denotes the number of omitted nodes. Further, in Tables 5-6 we report the relative errors given by the approach suggested in the Theorem 3 to compute the integrals $\int_{-1}^{\sigma_{n}} f_{i}(x) d x, i \in\{2,3\}$.

Finally, we have used the formula (3) to compute the integral

$$
I\left(f_{5} w_{\gamma}\right)=\int_{0}^{\infty} x^{10} e^{-2 x} d x
$$

with $\gamma=0$ and $f_{5}(x)=x^{10} e^{-x}$. In Table 7 we report the relative errors for different choices of the sequences $\left\{\tau_{n}^{\prime}\right\}_{n \in N}$ and $\left\{\tau_{n}\right\}_{n \in N}$. Here $n$ denotes the number of the gaussian nodes while the numbers in square brackets denote the numbers of the omitted nodes near 0 and sufficiently large, respectively.

All the numerical experiments are carried out with MATLAB/R2011a.

\section{Acknowledgement}

This work was supported by the Italian Research Council under department project ICT.P11 Modeling and Simulation of Complex Systems

\section{References}

[1] M.R. Capobianco, G. Criscuolo, Some remarks on the numerical computation of integrals on an unbounded interval, Numer. Algorithms, 45, 37-48 (2007).

[2] Z. Ditzian, D.S. Lubinsky, Jackson and smoothness theorems for Freud weights in $L_{p}(0<p \leq \infty)$, Constr. Approx., 13, 99-152 (1997).

[3] G. Freud, Orthogonal Polynomials, Pergamon Press, Akadémiái Kiadó, Budapest, (1971).

[4] W. Gautschi, Orthogonal Polynomials: Computation and Approximation, Clarendon Press, Oxford, (2004).

[5] W. Gautschi, Gauss quadrature routines for two classes of logarithmic weight functions. Numer. Algorithms, 55, 265277 (2010).

[6] G. Mastroianni, G. Monegato, Truncated Gauss-Laguerre quadrature rules. Recent trends in numerical analysis, , Adv. Theory Comput. Math., Nova Sci. Publ., Huntington, NY, 3, 213-221 (2001).

[7] G. Mastroianni, G. Monegato, Truncated quadrature rules over $(0, \infty)$ and Nyström-type methods, SIAM J. Numer. Anal., 41, 1870-1892 (2003).
[8] G. Mastroianni, G. Monegato, Truncated interpolation processes on unbounded intervals and their applications, Rend. Circ. Mat. Palermo, 55, 123-139 (2006).

[9] G. Mastroianni, G. Monegato, Some new applications of truncated Gauss-Laguerre quadrature formulas, Numer. Algorithms, 49, 283-297 (2008).

[10] G. Mastroianni, G. Monegato, Erratum: Some new applications of truncated Gauss-Laguerre quadrature formulas, Numer. Algorithms, 52, 507 (2009).

[11] G. Mastroianni, G. Monegato, Numerical integration of functions with a very small significant support, J. Comput. Appl. Math., 236, $4082-4089$ (2012).

[12] A.H. Stroud, Kwan-Wei Chen, Peano error estimates for Gauss-Laguerre quadrature formulas, SIAM J. Numer. Anal., 9, 333-340 (1972).

[13] G. Szegö, Orthogonal Polynomials, Amer. Math. Soc., Providence, R.I., 23, (1975).



Giuliana Criscuolo was born in Naples, Italy, on November 1955.

From 1980 to 1992, she worked within National Research Council in Italy. She is currently a Professor of Numerical Analysis in the Department of Mathematics and Applications, University of Naples Federico II, Italy, since 1992. Her current research interest include numerical analysis, orthogonal polynomials, approximation theory, numerical integration and numerical methods.

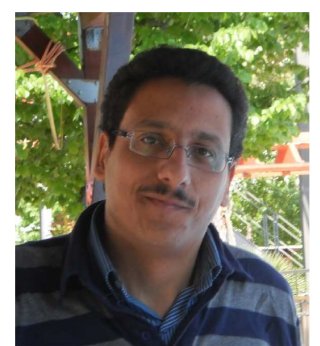

\section{Salvatore Cuomo}

is Assistant Professor in Numerical Analysis at University of Naples Federico II. His research field interests are in Mathematical models for applied sciences, Numerical Approximation theory and applications, Parallel and Distributed computing, Inverse problems arising from image analysis and Information Technology for automatic data processing in medicine and teaching environments $\mathrm{He}$ is referee and editor of mathematical journals. 